Supporting information

\title{
Reusability Comparison of Melt-Blown vs. Nanofiber Face Mask Filters for Use in the Coronavirus Pandemic
}

Sana Ullah ${ }^{\ddagger \ddagger}$, Azeem Ullah ${ }^{\alpha \ddagger}$, Jaeyun Lee $^{\beta \ddagger}$, Yeonsu Jeong ${ }^{\beta^{\ddagger}}$, Motahira Hashmi $^{\alpha}$, Zhu Chunhong ${ }^{\mathfrak{f}}$, Kye Il $\mathrm{Joo}^{\beta}$, Hyung Joon Cha ${ }^{\beta *}$, and Ick Soo Kim ${ }^{\alpha *}$

${ }^{\alpha}$ Nano Fusion Technology Research Group, Institute for Frontier Fibers, Shinshu University, Ueda campus, Nagano, 386-0017, Japan

${ }^{\beta}$ Department of Chemical Engineering, Pohang University of Science and Technology, Pohang 37673, Korea

${ }^{£}$ Faculty of Textile Science \& Technology, Shinshu University, Ueda campus, Nagano, 3860017, Japan

*Corresponding authors:

Hyung Joon Cha, hjcha@postech.ac.kr; Ick Soo Kim, kim@shinshu-u.ac.jp

${ }^{\ddagger}$ S. Ullah, A. Ullah, J. Lee, and Y. Jeong have equal contribution in this research. 
Scanning electron microscopic (SEM) images are shown in Figure S1, which represents nontreated, ethanol treated (spraying and dipping respectively) MB and NF filters. It was observed that there was not any considerable change in morphology of both types of filters after irrespective of treatment method.
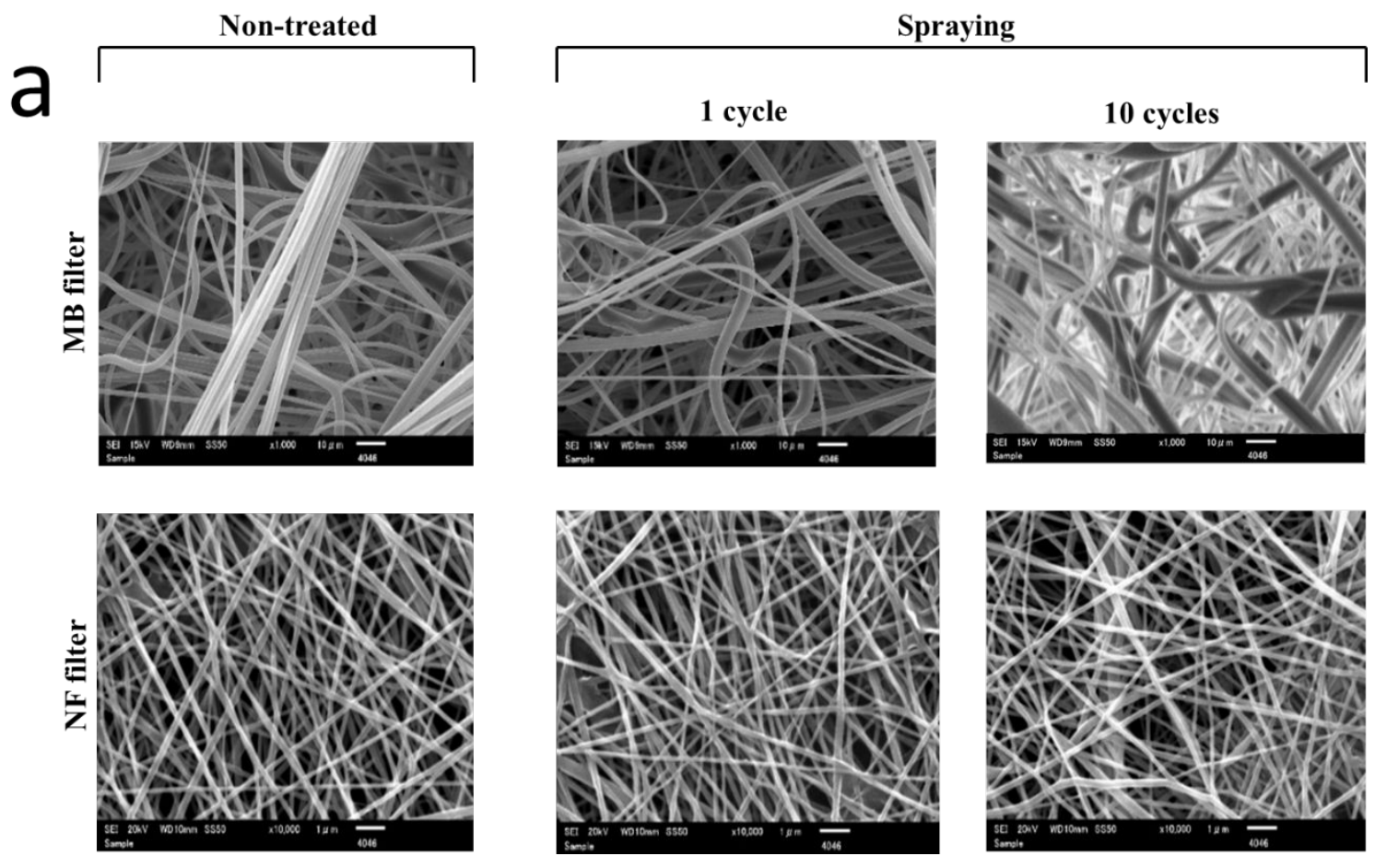

Dipping
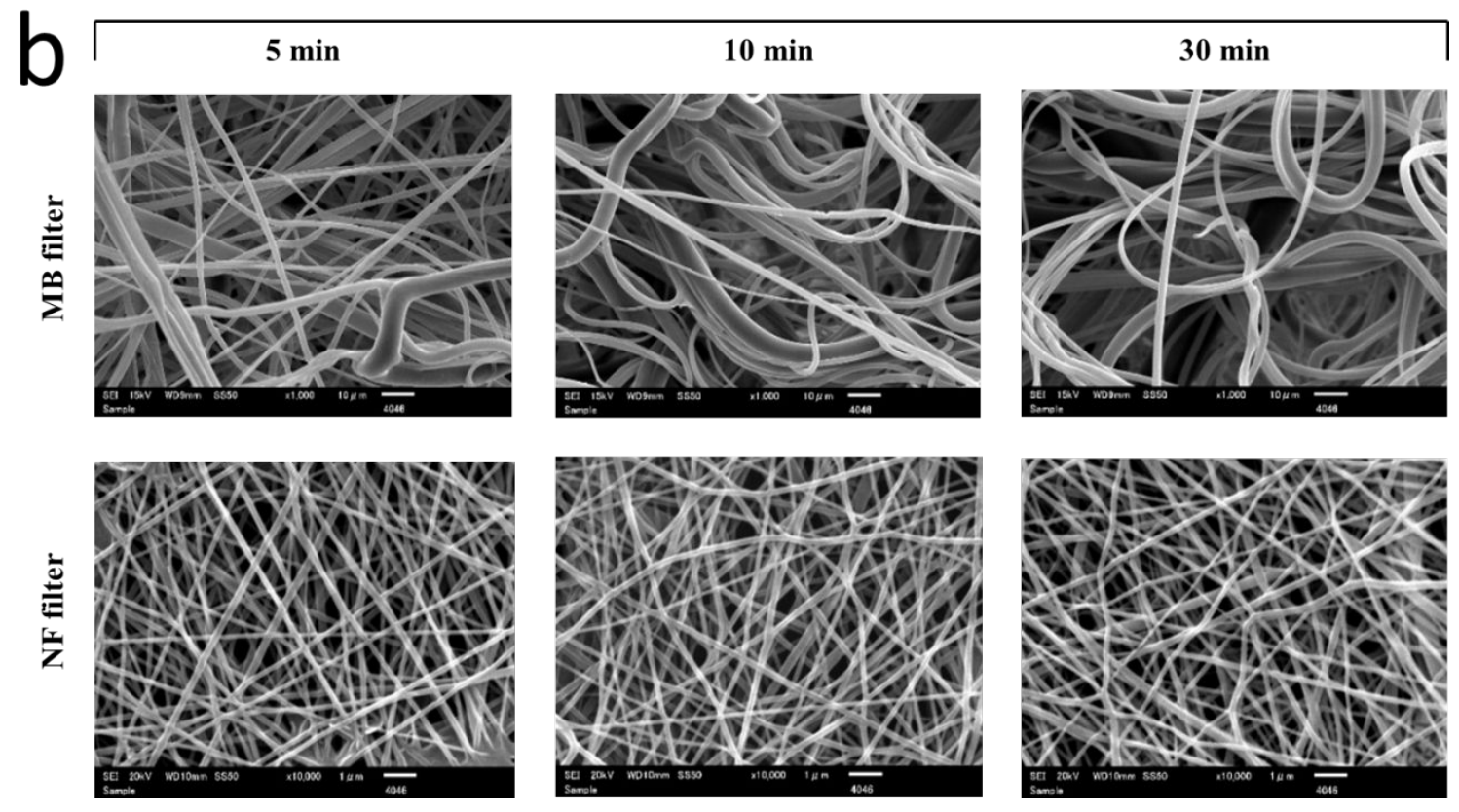

Figure S1 (a) Magnified SEM images of MB and NF filters (before and after treatment (ethanol spraying)), (b) SEM images of MB and NF filters after dipping treatment 